\title{
Journal of Medicine and Therapeutics
}

\section{Editorial}

\section{Epigenetics and its role in carcinogenesis}

\author{
Jibran Sualeh Muhammad* \\ Department of Basic Medical Sciences, College of Medicine, University of Sharjah, Sharjah, UAE
}

Epigenetics is a well-known mechanism playing a significant role in cancer development. It is characterized by stable and heritable changes in the function of genes which occur without any alteration to the DNA sequence and includes DNA methylation, histone modification, and RNA interference by microRNAs. These epigenetic modifications can be stable to maintain the specific lineage of cells or dynamic to respond to developmental and environmental signals. Alterations in the epigenetic state of the cell can lead to aberrancies in phenotype which are then passable to daughter cells [1].

DNA methylation in the CpG Islands (CGI) of the gene promoter region is one of the best known and extensively studied epigenetic modification. Methylation of several tumor suppressor gene promoters is responsible for their silencing and thus potentially sustains carcinogenesis. Similarly, histone de-acetylation can lead to oncogene activation. miRNA are small (18-20 nucleotides) non-coding RNA fragments capable of inhibiting other m-RNA, ultimately altering the balance in oncogene and tumor suppressor gene expression. It has been shown that growth of several tumor types can be stimulated by epigenetic changes in various phases of carcinogenesis, and drugs able to interfere with these mechanisms can have a positive impact on tumor progression. As matter of fact, dynamic epigenetic changes can be reversed by epigenetic inhibitors [2]

Recently, we published a study in the International Journal of Cancer which showed that methylation silencing of an autophagy related gene by Helicobacter pylori infection leads to carcinogenic transformation in gastric epithelial cells [3]. This study was a collaborative project between researchers from The Aga Khan University, University of Toyama, and the National Cancer Institute, Tokyo. We showed that MAP1LC3Av1 was specifically methylation-silenced in $H$. pylori-infected non-cancerous and cancerous gastric mucosae. The inactivation of map $1 l c 3 a$, a rat homolog of the human MAP1LC3Av1, disrupts the autophagy pathway, thereby leading to carcinogenesis in gastric epithelial cells. We suggested that, MAP1LC3Av1 methylation and expression levels could be used as an early indicator of carcinogenic transformation of non-cancerous mucosae in $H$. pylori-infected gastric cancer patients. Chemoprevention may also be achieved against gastric cancer associated with $H$. pylori using by de-methylating agents in future. Although we don't see many cases of $H$. pylori-induced gastric cancer among Pakistani population, however, gastric cancer is amongst the top ten prevalent cancers with gradual rise to a maximum in the $7^{\text {th }}$ decade of life. This study will provide a basis to study the role of DNA methylation in relation to the development of the more prevalent caners. Similar studies are in pipeline by our research group to investigate the role of epigenetic modifications in association with commonly prevalent diseases and cancers in Pakistani population.

\section{References}

1. Esteller M (2008) Epigenetics in cancer. N Engl J Med 358:1148-1159. [Crossref]

2. Sharma S, Kelly TK, Jones PA (2010) Epigenetics in cancer. Carcinogenesis 31: 2736. [Crossref]

3. Muhammad JS, Nanjo S, Ando T, Yamashita S, Maekita T, et al. (2017) Autophagy Impairment by Helicobacter pylori-induced Methylation Silencing of MAPILC3AvI Promotes Gastric Carcinogenesis. Int J Cancer 140: 2272-2283. [Crossref]
Copyright: (C)2017 Muhammad JS. This is an open-access article distributed under the terms of the Creative Commons Attribution License, which permits unrestricted use, distribution, and reproduction in any medium, provided the original author and source are credited.
Correspondence to: Dr. Jibran Sualeh Muhammad MBBS, Ph.D., Assistant Professor, Department of Basic Medical Sciences, College of Medicine, University of Sharjah, Sharjah, UAE, E-mail: dr.jibran@live.com

Received: May 10, 2017; Accepted: May 16, 2017; Published: May 18, 2017 